\title{
"Effects of resolution on the relative importance of numerical and physical horizontal diffusion in atmospheric composition modelling" published in Atmos. Chem. Phys., 10, 2737-2743, doi:10.5194/acp-10-2737-2010, 2010
}

M. D'Isidoro, A. Maurizi, and F. Tampieri

CNR-ISAC, via Gobetti 101, 40129 Bologna, Italy

Unfortunately, a typing error was noticed in the acknowledgements to the online publication and the final version should be as follows:

Acknowledgements. The authors would like to thank Piero Malguzzi for his valuable support. This work was supported by the FP6 IP GEMS and FP7 CITYZEN and the Special Project "Impatto degli "hot spot" sui cambiamenti climatici a scala regionale" financed in the frame of the activities of the Centro Euro Mediterraneo per i Cambiamenti Climatici (CMCC). 